Classification

Physics Abstracts

$87.90+\mathrm{y}-87.25-\mathrm{a}-87.20-\mathrm{i}$

\title{
Biological electron energy loss spectroscopy: the present and the future
}

\author{
Richard D. Leapman $\left({ }^{1}\right)$ and S. Brian Andrews $\left({ }^{2}\right)$ \\ ( $\left.{ }^{1}\right)$ Biomedical Engineering and Instrumentation Program, NCRR, National Institutes of Health, \\ Bethesda, Maryland 20892, U.S.A. \\ $\left({ }^{2}\right)$ Laboratory of Neurobiology, NINDS, National Institutes of Health, Bethesda, Maryland 20892, \\ U.S.A.
}

(Received December 06, 1990; accepted March 26, 1991)

\begin{abstract}
The current state of electron loss spectroscopy (EELS) techniques and applications in biology is briefly reviewed. The field-emission scanning transmission electron microscope (STEM) equipped with a parallel detection spectrometer can localize and quantitate physiologically and biochemically relevant concentrations of many important elements in specimens prepared by suitable low-temperature techniques. The energy-filtering TEM is a complementary instrument, well suited for mapping locally high concentrations of elements over wider sample regions. With the STEM, nearly single-atom sensitivity can be achieved in the analysis of individual macromolecular assemblies although the spatial resolution is limited by radiation damage. Other STEM energy loss techniques that appear to be feasible include: low-dose elemental mapping of periodic structures; elemental labelling of organic and organometallic compounds in cells; thickness measurement of hydrated proteinb crystals, and measurement of water content in subcellular compartments.
\end{abstract}

\section{Introduction.}

Biological specimens often challenge the limits of microanalysis because of the low concentrations of elements that they contain. In proteins and nuclei acids, in assemblies of proteins and in cell organelles, the atomic fractions of biologically important elements such as phosphorus, calcium and other metals are typically in the range of only $\sim 10^{-5}$ to $10^{-2}$. Energy-dispersive $\mathrm{x}$ ray spectroscopy (EDXS) is the technique most commonly used for biological microanalysis: for these applications a high sensitivity and capability for quantitation have been demonstrated [1-3]. Electron energy loss spectroscopy (EELS) has generally been considered a specialized technique most useful for detecting high concentrations of light elements. The small signal/background that is encountered in EELS microanalysis clearly imposes demanding requirements for instrumentation and data processing. In many cases the increased sensitivity of parallel detection becomes essential for obtaining useful results [4-6]. However, such a capability has become available only in the past few years, so the full potential of EELS applications in biology has yet to be explored [7]. 
The success of microanalytical experiments strongly depends on sample preparation. For example, detection of diffusible elements in cells relies on cryosectioning of rapidly frozen tissue; often the resulting samples are too thick for EELS analysis. In other situations radiation damage limits the total dose tolerated by the sample so that counting statistics determine sensitivity [8]. Despite the limitations imposed by preparative considerations, substantial progress has been made in recent years. Our purpose here is to outline the current state of EELS in biology and to indicate some future directions that the field may take.

\section{STEM-PEELS vs. energy-filtering TEM.}

Two types of instrumentation are currently being used to apply EELS in biology. One is the scanning transmission electron microscope (STEM) equipped with a magnetic-sector spectrometer $[6,9]$; the other is the TEM combined with an energy-filter (EFTEM), either a Castaing-Henry magnetic-sector/ electrostatic mirror [10-12] or a purely magnetic-sector $\Omega$-filter [13,14]. To appreciate the capabilities and limitations of biological EELS it may be helpful to summarize some of the pros and cons of STEM and EFTEM approaches.

In either microscope, EELS analysis generally requires a high electron dose, typically $10^{8} \mathrm{e} / \mathrm{nm}^{2}$; such a dose guarantees significant mass loss. Nevertheless, additional radiation damage can still be a limiting factor for many types.of specimens, and so it is desirable to minimize specimen exposure. In this regard, it is clear that STEM in combination with parellel detection offers the advantage if information from more than one energy channel is required. Multiple exposures to the electron beam are necessary with the energy filter but only one exposure is required per image point with the STEM. Spectra covering a range of energies are in fact required for the important application of elemental mapping, where several channels must be collected in the vicinity of a core edge in order to model the background $[15,16]$.

The main advantage of the energy-filtering TEM is the parallel nature of the image formation. Data from a very large number of "pixels" can be acquired simultaneously and recorded either electronically or on photographic film. Future advances in solid state camera technology should improve sensitivity, quantitation and speed for recording filtered images; slow-scan CCD cameras are particularly promising because of their high detective quantum effeciency (DQE), linearity and dynamic range [17]. Comparable spatial resolution can be achieved in the STEM provided it is equipped with a high brightness field-emission source. However, the total acquisition time for a STEM EELS map is generally much greater than with energy-filtering because the data must be acquired pixel by pixel and the total probe current is relatively small, typically $1 \mathrm{nA}$ [18]. As an example, if the pixel dwell time is $50 \mathrm{~ms}$, a $256 \times 256$ map takes one hour to record. The length of the acquisition time can be a severe limitation, necessitating correction for sample drift.

On the other hand the STEM with parallel EELS provides the opportunity for spectrum imaging [19], i.e. acquisition of an entire spectrum at each pixel. Although enormous quantities of data are generated, spectrum imaging permits great flexibility in off-line processing. In biological microanalysis it allows us to detect physiologically relevant concentrations of elements, such as calcium, that produce weak core edges on large backgrounds (signal/background $<10^{-3}$ ) $[5,6,9]$. Quantitation and accuracy of analysis is also facilitated in the STEM approach. For example, multiple least-squares fitting can be performed at each pixel in an elemental map [20,21]. In addition only the STEM allows simultaneous acquisition of EELS and energy-dispersive x-ray (EDXS) data; this capability can be very important for many biological applications where the distributions of several elements are required. An example is the detection of potassium, where the limits are much better for EDXS than for EELS because of overlap of the $\mathrm{K} \mathrm{L}_{23}$ and $C \mathrm{~K}$ edges.

There are applications where the energy-filter may offer advantages. Consider, for example, 
detection of an element present at high concentrations over an extended sample area, such as ferritin molecules ( $\sim 30 \mathrm{wt}-\%$ iron) in a thin plastic embedded section. In this case the background estimation is relatively straightforward and a meaningful map can be recorded from a large number of pixels. The EFTEM may also be useful in certain pathology applications where high local elemental concentrations exist.

Perhaps the most useful biological application of the EFTEM is for increasing contrast in thick biological sections by filtering out the inelastic scattering contribution in the bright-field image. The improvement is due to the absence of chromatic aberration in the resulting zero-loss image. It is also possible to make use of the inelastic dark-field image to improve contrast in unstained plastic sections [22-24]; such images only gives information about morphology and not elemental distributions.

\section{Information from the core-loss spectrum.}

3.1 MEASUREMENT OF BOUND ELEMENTS IN CONVENTIONAL EMBEDDED SECTIONS. - EELS can be most easily performed on thin plastic-embedded sections which can routinely be cut to a thickness of $<50 \mathrm{~nm}$. Microanalytical sensitivity is then not compromised by the effects of plural inelastic scattering. Unfortunately for most important applications, conventional embedding procedures drastically alter the composition of the sample and prevent us from obtaining useful data. There are some exceptions to this rule, such as the use of low temperature freeze-substitution techniques to help preserve elemental distributions in plastic sections, and immunolabelling and cytochemical methods [25]. Most often, however, we are obliged to use specimens that have not been fixed and embedded in a supporting medium, and which therefore are stabilized only by low temperatures.

3.2 MEASUREMENT OF IONS AND BOUND ELEMENTS IN DEHYDRATED CRYOSECTIONS. - Microanalysis of freeze-dried cryosectioned tissue is normally performed with $\mathrm{X}$-ray spectroscopy [13]. In the past few years, however, it has been demonstrated that EELS can provide improved detectability for certain important elements such as calcium [6], phosphorus, and sodium [26] in an organic matrix. The improvement is approximately a factor of three for calcium and phosphorus, and more for sodium. In order to achieve this level of sensitivity it is essential to use parallel detection and to process the spectra appropriately. Thus, first or second difference acquisition can be used to remove channel-to-channel gain variations in the detector, while also increasing visibility of the signal. Quantitation can then be achieved by fitting reference spectra obtained from suitable standards [6,9] using a multiple least-squares procedure.

Certain elements such as potassium are invariably better analyzed using $\mathrm{x}$-rays and it is therefore desirable to perform EELS and EDX simultaneously. One main difficulty with EELS analysis of dried cryosections is the large local variation in sample thickness that is normally present. In addition, at $100 \mathrm{keV}$ beam energy plural inelastic scattering can significantly reduce sensitivity. It would therefore be advantageous to operate at 200 or $300 \mathrm{keV}$ beam energy in order to increase the inelastic mean free path.

3.3 ANALYSIS OF MACROMOLECULES AND MACROMOLECULAR ASSEMBLIES. - The combination of field-emission STEM and parallel detection EELS [27] allows the detection of specific bound elements within individual macromolecules prepared by direct freezing and freeze-drying $[28,29]$. In order to achieve the required sensitivity of a few atoms an extremely high electron dose $\left(\sim 10^{9}\right.$ to $\left.10^{10} \mathrm{e} / \mathrm{nm}^{2}\right)$ is required; this clearly will entail terminal radiation damage and "knock-on" damage may also occur. However, the mass distributions and molecular weights of 
the freeze-dried structures can first be characterized at low dose $\left(\sim 10^{3} \mathrm{e} / \mathrm{nm}^{2}\right)$ by digital darkfield mass mapping [30-32]. The probe current is then increased by several orders of magnitude for elemental analysis. For a single molecule, the necessary counting statistics are achieved in spectra acquired for about $100 \mathrm{~s}$. It is important to optimize extraction of the signal using methods such as first or second difference acquisition, and multiple least squares fitting [6,26]. Results indicate that a remarkably high sensitivity is attainable. For example, the 320 copper atoms in a single hemocyanin molecule $(M W=8 \mathrm{Md}, 0.3 \mathrm{wt}-\% \mathrm{Cu})$ can be easily detected [29]. Indeed, detection of the four iron atoms in a single molecule of hemoglobin $(M W=65 \mathrm{kd}, 0.4 \mathrm{wt}-\% \mathrm{Fe})$ has been demonstrated provided extremely high doses are used [29]. Small numbers of phosphorus atoms can also be detected in macromolecular assemblies; this is illustrated by the spectrum in figure 1 obtained from a $\sim 10-\mathrm{nm}$ size area in the center of a tobacco mosaic virus (a rod-shaped structure of length $300 \mathrm{~nm}$, width $18 \mathrm{~nm}$ and $M W=39 \mathrm{Md}$ ).

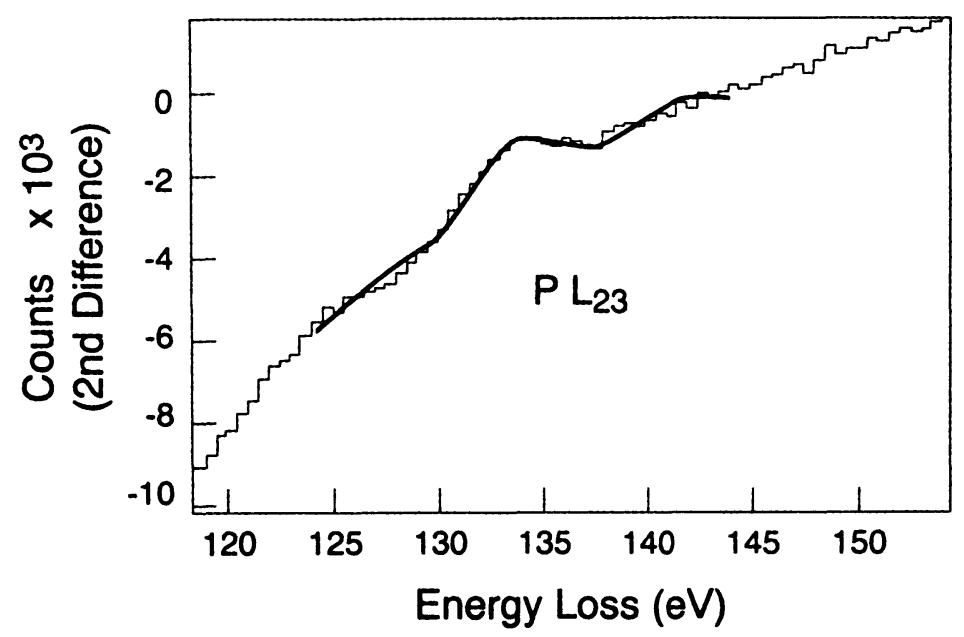

Fig. 1. - Second-difference EELS spectrum from 10nm-size region at center of tobacco mosaic virus. Phosphorus $\mathrm{L}_{23}$ signal is due to single strand of RNA containing approximately 200 phosphorus atoms. MLS fit is superimposed (solid line). Note phosphorus signal in not visible in raw spectrum because signal is obscured by channel-to-channel gain variations of the parallel-detector array.

The signal, as estimated from scattering cross sections [16], is consistent with the known structure of the virus, i.e., a segment of single-stranded RNA containing 200 phosphorus atoms at $\sim 0.9$ wt $-\%$ concentration. Such low levels of phosphorus are not normally detectable with EELS because of the small signal/background ratio, and the result is only possible with parallel detection. An appealing application for phosphorus detection is the direct determination of the phosphorylation state of cytoskeletal and regulatory proteins. Even though it is impossible to attain a spatial resolution much smaller than the dimension of the molecule (or $\sim 10 \mathrm{~nm}$ ), the combination of molecular weight characterization by low-dose, dark-field STEM and quantitation of specific bound atoms by high-dose EELS may prove to be a useful technique.

3.4 DETECTION OF LABELlED COMPOUNDS. - The possibility of using EELS to localize fluorine -labelled compounds in specific compartments of cells was first suggested by Isaacson and Johnson [33]. Due to previous limitations in instrumentation there have not yet been any convincing applications of this approach. However, recent data on the stability of organic compounds 
containing $\mathrm{F}$ atoms on aromatic rings or in trifluoromethyl groups [34] - especially when stabilized against radiation-induced fluorine loss by cooling to liquid nitrogen temperatures - provides new evidence that such experiments may in fact be feasible. For example, data on fluorinated derivatives of the hormone epinephrine indicate that physiological concentrations of the compound should be detectable in secretory granules of adrenal chromaffin cells [18]. Atoms other than fluorine should also be useful for labelling organic compounds but care must be taken to preserve biological activity. Eventually it may be possible to characterize neuronal synapses by localizing labelled neurotransmitters and their precursors within the synaptic vesicles or other cellular compartments of presynaptic terminals.

EELS promises to be a useful technique for localizing immunolabelled antigens in cells. Indeed, ferritin molecules - a historically common antibody label - are easily detected in the EELS spectrum by the iron $\mathrm{L}_{23}$ edge. Ferritin can usually be identified in micrographs without resort to microanalysis, and in any case ferritin labeling has largely been supplanted by colloidal gold techniques. However, empirical "energy tuning" in the EFTEM provides enhanced contrast of unstained gold-labeled specimens [23], which is especially valuable for visualizing the smaller (1and 5-nm) gold particles that are favored for enhanced penetration of label. In addition, EELS might facilitate multiple-labelling experiments where different antigens are recognized by antibodies contributing qualitatively or quantitatively different elemental signals. Recently, the boron $\mathrm{K}$-edge signal has been used to localize boronated protein A labelled antibodies bound to specific antigens in cells [35]. Because an element like boron provides the opportunity to fashion small, low molecular weight labels, it offers the prospect of immunoreagents with improved tissue penetration properties.

3.5 LOW-DOSE EELS MAPPING. - It has been proposed that elemental mapping at low dose should be possible by averaging the characteristic core loss signal over many identical structures, such as a periodic crystalline array of macromolecules (e.g. reconstitued membranes) [36]. In the case of a filamentous structure, the radial elemental distribution can be determined by averaging along its length. Such experiments are only feasible if the spectrum can be read out very rapidly at each pixel $(\sim 100 \mu \mathrm{s})$ and if single-electron detection is possible, i.e. high DQE at low electron fluxes. Although the photodiode array detector is highly optimized for most microanalytical applications it does not satisfy the requirements for low dose measurements and this has led to a number of proposals for alternative devices. An ion-implanted silicon array exposed directly to the electron beam has been reported by Haider [37], a ten channel silicon diode is under development by Krivanek et al. [38], and Reichelt and Engel [36] have developed a system based on three separate scintillators and photomultipliers. These new optimized detectors in combination with signal averaging techniques should encourage experiments to localize nucleic acids, proteins and lipids in ordered assemblies at nanometer resolution using low-dose $\left(10^{3}-10^{4} \mathrm{e} / \mathrm{nm}^{2}\right)$ mapping of phosphorus and nitrogen.

\section{Information from the low-loss spectrum.}

4.1 WATER CONTENT OF CELLULAR ORGANELLES. - The mass thickness of a thin sample can be determined at very low dose by measuring the fraction of transmitted electrons that are inelastically scattered [39] and this can lead to a variety of useful information in biological specimens. In cell physiology, the water concentration of subcellular compartments is an important quantity - one that has been virtually impossible to measure directly on the subcellular scale. It is possible to estimate the water content of organelles by measuring the fraction of inelastically scattered electrons in hydrated and subsequently dehydrated cryosections. Some assumptions are necessary 
concerning the inelastic cross section per unit mass for the organic and aqueous components of the cell, but the main errors are due to shrinkage of the section on drying [18]. It may prove possible to extract the information directly from spectrum-images of samples in their hydrated state by taking advantage of differences in the low-loss spectral shape as a function of water content, thus eliminating the error due to shrinkage.

4.2 THICKNESS OF HYDRATED PROTEIN CRYSTALS. - Three-dimensional electron microscopy of hydrated ice-embedded protein crystals depends on knowledge of the crystal thickness in order to combine correctly images recorded at different tilts. The energy loss spectrum recorded at low dose from a small ( $\sim 100 \mathrm{~nm}$ diameter) region allows us to determine the number of unit cells in the crystal thickness [40]. This method depends on the difference in the inelastic mean free path for the hydrated crystal and water. Preliminary data obtained from hydrated catalase [41] demonstrate that the method is sufficiently sensitive to measure the exact number of unit cells in specimens less than $100 \mathrm{~nm}$ thick.

4.3 Z-CONTRAST AND OTHER NOVEL IMAGING MODES. - By combining the integrated energyloss (inelastic), zero-loss (unscattered) and annular dark-field (elastic) signals in the STEM we can estimate the mean atomic number of different compartments in a cell [42-44]. This information, in turn, permits an estimate of the composition in terms of protein, nucleotide, and lipid content. The simplest approach is to take the ratio of elastic and inelastic signals which is approximately proportional to the atomic number (referred to as $Z$ - contrast). It has been demonstrated, however, that this result is only valid in the limit of very thin samples [44,45], so it is practical to obtain useful data only from thin, unstained, plastic sections. The mean $Z$ of the embedding material can be chosen to enhance the visibility of particular components, such as lipids or nucleotides. It is possible to refine the method by combining signals so that the effects of plural scattering are minimized. Haider [46] has placed the annular dark-field detector after the spectrometer to obtain energy-filtered, dark-field images in the STEM; it is then possible to obtain pure $Z$-contrast in samples of finite thickness.

\section{Conclusions and future directions.}

Instrumentation for electron energy loss spectroscopy has now advanced to a stage where interesting experiments in biology have become feasible. The energy-filtering TEM and the STEM with parallel EELS each has advantages for different types of applications.

EFTEM is often the method of choice when elemental concentrations are locally high. On the other hand, physiologically relevant concentrations of elements, such as calcium, sodium and phosphorus can be better detected in STEM using a parallel detection spectrometer, while simultaneous $x$-ray data can be recorded for other elements. The limitations in applying EELS to biology often lie with difficulties in specimen preparation rather than with the spectroscopy, particularly when cryosectioning is required. In the future we may expect attempts to localize specific organic compounds in subcellular compartments using fluorinated compounds and other labelling approaches. The combination of low-dose STEM molecular weight determination (mass mapping) and high-dose elemental analysis of macromolecular assemblies is feasible at almost single-atom sensitivity. However, the task of obtaining elemental distributions at low-dose is considerably more complicated and depends on novel detection devices with parallel read-out and single-electron sensitivity, as well as averaging procedures for optimizing signal/noise. In the future we may also expect use of the low-loss spectrum to obtain information about the water content of organelles and to determine the thickness of protein crystals required for three-dimensional 
structure determination. Although EELS is still not used routinely in the study of biological samples, its potential for obtaining many types of important information has long been recognized. Soon, this potential should be realized.

\section{References}

[1] SOMLYO A.P. and SHUMAN H., Ultramicrosc. 8 (1982) 219-234.

[2] SOMLYO A.P., BOND M. and SOMLYO A.V., Nature (London) 314 (1985) 622-625.

[3] ANDREWS S.B., LEAPMAN R.D., LANDIS D.M.D. and REESE T.S., Proc. Natl. Acad. Sci. USA 85(1988) 1682-1985.

[4] SHUMAN H., Ultramicrosc. 6 (1981) 163-168.

[5] SHUMAN H. and KRUIT P., Rev. Sci. Instrum. 56 (1985) 231-239.

[6] SHUMAN H. and SOMLYO A.P., Ultramicrosc. 21 (1987) 23-32.

[7] KRIVANEK O.L., AHN C.C. and KEENEY R.B., Ultramicrosc. 22 (1987) 103-116.

[8] ISAACSON M.S., In: Principles and Techniques of Electron Microscopy. VII, M.A. Hayat (Ed.) (Van Nostrand-Reinhold, New York, 1977) 1-78.

[9] LEAPMAN R.D., In: Electron Probe Microanalysis- Applications in Biology and Medicine, K. Zierold and H.K. Hagler (Eds.), Springer Series in Biophysics, (Springer-Verlag, Berlin) 4 (1989) 113-125.

[10] Castaing R. and Henry L., C.R. Acad. Sci. Paris 255 (1962) 76-78.

[11] OTTENSMEYER F.P. and ANDREW J.W., J. Ultrastruct. Res. 72 (1980) 336-348.

[12] OTTENSMEYER F.P., J. Ultrastruct. Res. 88 (1984) 121-134.

[13] ZANCHI G., PEREZ J.P. and SEVELY J., Optik 43 (1975) 495-501.

[14] Krahl D., Herrmann K.-H. and Kunath W., In: Electron microscopy -1978, 9th Int. Cong., J.M. Sturgess Ed. (Microscopical Society of Canada, Toronto) 1 (1978) 42-43.

[15] Jeanguillaume C., CollieX C. and TRebBia P., Ultramicroscopy 3 (1978) 137-142.

[16] EgerTon R.F., In: Electron Energy Loss Spectroscopy in the Electron Microscope, (Plenum, New York, 1986).

[17] KRIVANEK O.L. (1990) (private communication).

[18] LEAPMAN R.D. and ORNBERG R.L., Ultramicroscopy 24 (1988) 251-268.

[19] JEANGUILlaUME C. and COLLIEX C., Ultramicroscopy 28 (1989) 252-257.

[20] Shuman H., Chang C.-F., Buhle E.L.Jr. and Somlyo A.P., Ann. N.Y. Acad. Sci. 483 (1986) 295-310.

[21] HunT J., In: Proceedings of the 47th Annual Meeting of EMSA, G.W. Bailey Ed. (San Francisco Press, San Francisco, 1989) 398-399.

[22] WAGNER H.-J., Ultramicroscopy 32 (1990) 42-47.

[23] OZEL M., PAULI G. and GELDERBLOM H.R., Ultramicroscopy 32 (1990) 35-41.

[24] WAGNER R.C. and CHEN S.-C., J. Histochem. Cytochem. 38 (1990) 275-282.

[25] Livesey S.A., DEl CAMPO A.A., GRIFFEY E.S., OHLMER D., SCHIFANI T. and ChIOVETTI R., In: Proceedings of the 47th Annual Meeting of EMSA, G.W. Bailey Ed. (San Francisco Press, San Francisco, 1989) 242-243.

[26 LEAPMAN R.D. and HUNT J.A., Microsc. Microanal. Microstruct. 2 (1991).

[27] KrivaneK O.L., Paterson J.H. and Poppa H.R., In: Proceedings of the 47th Annual Meeting of EMSA, G.W. Bailey Ed. (San Francisco Press, San Francisco, 1989) 410-411.

[28] LEAPMAN R.D. and ANDREWS S.B., J. Microsc. 161 (1991) 3-19.

[29] LEAPMAN R.D. and ANDREWS S.B. (1991) (in press).

[30] WAll J.S. and HAINFELD J.F., Ann. Rev. Biophys. Biophys. Chem. 15 (1986) 355-76.

[31] ENGEL A., Ultramicrosc. 3 (1978) 273-281.

[32] MOSESSON M.W., HAINFELD J., WALl J. and HASCHEMEYER R.H., J. Mol. Biol. 153 (1982) 695-718.

[33] ISAACSON M.S. and JOHNSON D., Ultramicroscopy 1 (1975) 33-52.

[34] CiliaX B.J., KiRK K.L. and Leapman R.D., In: Proc. 12 th Internat. Cong. Electron Microscopy, Seattle L.D. Peachey, D.B. Williams Eds. (San Francisco Press, San Francisco, CA) 2 (1990) 408409.

[35] Bendayan M., BARTh R.F., GRingas D., LONDONo I., Robinson P.T., AlaM F., AdAMS D.M. and MATTIAZZI L., J. Histochem. Cytochem. 37 (1989) 573-580. 
[36] Reichelt R., ENGEl A. and Leapman R.D., In:Proc. 11th Internat. Cong. Electron Microscopy, Kyoto T. Imura, S. Maruse, T. Suzuki Eds. 1 (1986) 33-34.

[37] HAIDER M., Boulin Ch. and EPSTEIN A., Inst. Phys. (U.K.) Conf. Ser. No. 931 (1988) 123-124.

[38] KrivaneK O.L., Delby N., Gubbens A.J., Kundmann M.K., Leber M.L., RAY D.A. and Truong K.V., In: Proc. 12 th Internat. Cong. Electron Microscopy Seattle, L.D. Peachey, D.B. Williams Eds. (San Francisco Press, San Francisco, CA) 2 (1990) 76-77.

[39] LEAPMAN R.D., FIORI C.E. and SWYT C.R., J. Microsc. 133 (1984) 239-253.

[40] REZ P. (1990) (unpublished results).

[41] CHIU W. and LEAPMAN R.D. (1991) (unpublished results).

[42] COlliex C., JeANGUillaume C. and MORY C., J. Utrastruct. Res. 88 (1984) 177-206.

[43] Carlemalm E., CollieX C. and Kellenberger E., In: Advances in Electronics and Electron Physics, L. Marton Ed. (Academic Press Inc., New York, 1985) 269-334.

[44] REICHELT R. and ENGEL A., Uttramicroscopy 13 (1984) 279-294.

[45] EGERTON R.F., Ultramicroscopy 10 (1982) 297-300.

[46] HAIDER M., Ultramicroscopy 28 (1989) 240-247. 